\title{
Region Growing Based nonlocal filtering for InSAR
}

\author{
Gerald Baier*, Xiao Xiang Zhu*† \\ * Remote Sensing Technology Institute (IMF), German Aerospace Center (DLR), \\ Oberpfaffenhofen, 82234 Wessling, Germany \\ $\dagger$ Helmholtz Young Investigators Group SiPEO, Technische Universtiät München (TUM), \\ Arcisstr. 21, 80333 Munich, Germany
}

\begin{abstract}
This paper proposes a nonlocal filter variant that replaces the conventional static search window of nonlocal InSAR filters with an adaptive region growing based search window. The region growing approach has the allure that it preselects only similar pixels for the averaging process and that it may find a larger number of statistically homogeneous pixels than a traditional, fixed search window. A Monte-Carlo simulation shows the possible benefits that could be realized with the region growing approach for InSAR filtering. The proposed method is also experimentally evaluated for rural and urban test sites.
\end{abstract}

\section{INTRODUCTION}

The nonlocal filtering concept was first introduced for optical images by Buades et al. in [1], and exploits that redundancy and repetition of structures and features are present in almost every natural image. Deledalle et al. [2] later adapted this concept to the noise statistics of interferometric synthetic aperture radar (InSAR). It has been widely demonstrated that the nonlocal means filter can significantly reduce noise while well preserving fine details, making it possible to, for example, produce InSAR-based digital elevation models of yet unseen quality [3].

Yet, many pixels in the search window will not contribute greatly to the final output due to their indiscriminate selection. This paper presents how the ordinarily employed static search window of a nonlocal filter for InSAR can be replaced by an adaptive search window based on a region growing approach, exploiting the fact that many man-made and natural structures or textures exhibit a certain self-similarity. By starting from one or several initial seed pixels and iteratively extending the region to include the most appropriate, bordering pixels, region growing possibly produces a result of similar quality, without having to rely on the same exhaustive search of pixels in the search window. To the best of our knowledge, region growing for nonlocal filters was so far only introduced to optical images in [4]; a separate treatment for SAR images is justified, since region growing avoids some of the problems that are commonplace with InSAR images, such as the fringes of the topography or changes of the terrain which prevent traditional nonlocal filters from finding larger numbers of similar pixels.

\section{Nonlocal Filtering CONCEPT AND REgion GROWING}

Figure 1 elucidates the filtering process: for a target pixel (the central black pixel) the similarities to all pixels inside a search window (grey shaded area) are computed. To ensure that also structures and textures are taken into account, not only the pixels themselves, but also their surrounding areas (patches), depicted here by the bold black rectangles, are used for computing the similarities, which are then mapped into weights, usually by an exponential kernel, or an adaption thereof. The final filtered target pixel is computed via weighted means.

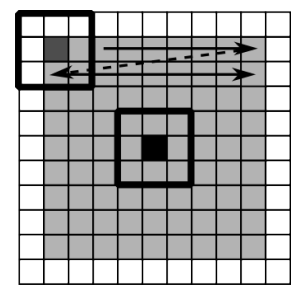

Fig. 1. nonlocal filtering concept

Basically the filtering procedure can be broken down into several steps:

1) A function that given the target pixel $\left(x_{t}, y_{t}\right)$ produces a list of pixels in the search window: $f_{\mathrm{sw}}:\left(x_{t}, y_{t}\right) \mapsto$ $\left[\left(x_{i}, y_{i}\right)\right]$

2) A function that given a list of pixels in the search window, computes a list of similarities between the patches centered around those pixels and the patch of the target pixel $f_{\text {sim }}:\left[\left(x_{i}, y_{i}\right)\right] \mapsto\left[\xi_{i}\right]$

3) A weighting kernel that computes a weight from a similarity, which is extended to work on lists $f_{\text {weight }}$ : $\left[\xi_{i}\right] \mapsto\left[\right.$ weight $\left._{i}\right]$

4) and finally the averaging function which given a list of weights computes an estimate of the pixel $f_{\text {average }}\left[\right.$ weight $\left._{\mathrm{i}}\right] \mapsto\left(\hat{x}_{t}, \hat{y}_{t}\right)$

The complete nonlocal filter can then be described as the composition of all previous functions

$$
f_{\mathrm{NL}}=f_{\text {average }} \circ f_{\text {weight }} \circ f_{\text {sim }} \circ f_{\mathrm{sw}}
$$

The idea of region growing is rather simplistic and its implementation straightforward, in essence only the function computing the search window pixels $f_{\mathrm{sw}}$ has to be adapted from an existing nonlocal filtering algorithm with a fixed rectangular search window.

\section{A. NLInSAR}

For our experiments we relied on the nonlocal InSAR filter NLInSAR introduced in [2], which computes an estimate of 
the reflectivity, the interferometric phase and the coherence given two single look complex (SLC) images.

NLInSAR is an iterative algorithm that utilizes both the original data (the master image's amplitude $A_{m}$, the slave image's amplitude $A_{s}$, and their interferometric phase $\varphi$ ) and the previous estimate (the reflectivity $\hat{r}$, the interferometric phase $\hat{\phi}$, and the coherence $\hat{\gamma}$ ) to arrive at the next estimate.

For comparing two patches of the original data the likelihood that these patches are noisy realizations of the same noiseless patch is computed. The likelihood of two noisy InSAR pixels $p_{1}$ and $p_{2}$ with the same parameter sets $\Theta_{i}=$ $\left\{r_{i}, \phi_{i}, \gamma_{i}\right\}$ for $i=1,2$ is given by [2]

$$
\begin{aligned}
p\left(p_{1}, p_{2} \mid \Theta_{1}\right. & \left.=\Theta_{2}\right) \\
& =\sqrt{\frac{C}{B}}\left(\frac{A+B}{A} \sqrt{\frac{B}{A-B}}-\arcsin \sqrt{\frac{B}{A}}\right)
\end{aligned}
$$

where

$$
\begin{aligned}
A & =\left(A_{m, 1}^{2}+A_{m, 2}^{2}+A_{s, 1}^{2}+A_{s, 2}^{2}\right) \\
B & =4\left(A_{m, 1}^{2} A_{s, 1}^{2}+A_{m, 2}^{2} A_{s, 2}^{2}\right. \\
& \left.+2 A_{m, 1} A_{s, 1} A_{m, 2} A_{s, 2} \cos \left(\varphi_{1}-\varphi_{2}\right)\right) \\
C & =A_{m, 1} A_{s, 1} A_{m, 2} A_{s, 2}
\end{aligned}
$$

The log-likelihood between two patches $\mathbf{p}_{1}$ and $\mathbf{p}_{2}$ is then computed as the sum over the corresponding pixel loglikelihoods

$$
\xi_{\mathrm{LL}}=\sum_{k} \log \left(p\left(\mathbf{p}_{1, k}, \mathbf{p}_{2, k} \mid \boldsymbol{\Theta}_{1, k}=\mathbf{\Theta}_{2, k}\right)\right)
$$

where $k$ denotes the $k$ th pixel in the patch.

The prior term, that the two parameter sets of the pixels, $\hat{\Theta}_{1}$ and $\hat{\Theta}_{2}$ are equal is exponentially proportional to their Kullback-Leibler divergence [2]

$$
\begin{aligned}
p\left(\hat{\Theta}_{1}=\hat{\Theta}_{2}\right) \propto & \exp \left\{\frac { 4 } { \pi } \left[\frac{\hat{r}_{1}}{\hat{r}_{2}}\left(\frac{1-\hat{\gamma}_{1} \hat{\gamma}_{2} \cos \left(\hat{\phi}_{1}-\hat{\phi}_{2}\right.}{1-\hat{\gamma}_{2}^{2}}\right)\right.\right. \\
+ & \left.\left.\frac{\hat{r}_{2}}{\hat{r}_{1}}\left(\frac{1-\hat{\gamma}_{1} \hat{\gamma}_{2} \cos \left(\hat{\phi}_{1}-\hat{\phi}_{2}\right.}{1-\hat{\gamma}_{1}^{2}}\right)-2\right]\right\}
\end{aligned}
$$

and conversely to the log-likelihood, the Kullback-Leibler divergence of two patches can be computed as

$$
\xi_{\mathrm{KL}}=\sum_{k} \log \left(p\left(\hat{\boldsymbol{\Theta}}_{1, k}=\hat{\boldsymbol{\Theta}}_{2, k}\right)\right)
$$

The similarity measure between two patches then consists of the tuple $\xi=\left(\xi_{\mathrm{LL}}, \xi_{\mathrm{KL}}\right)$, which is passed to the weighting kernel for computing the weight:

$$
w_{i}=\exp \left\{-\frac{\xi_{\mathrm{LL}}}{h}+\frac{\xi_{\mathrm{KL}}}{T}\right\}
$$

where $h$ and $T$ are smoothing parameters. There is one minor additional smoothing step for the weights, which for the sake of brevity is left out, the interested reader is referred to [2]. The final estimates are then computed by

$$
\begin{aligned}
\hat{r}_{t} & =\frac{a}{N} \\
\hat{\phi}_{t} & =-\arg x \\
\hat{\gamma}_{t} & =\frac{|x|}{a}
\end{aligned}
$$

$$
\begin{aligned}
a & =\frac{1}{2} \sum_{i} w_{i}\left(A_{m, i}^{2}+A_{s, i}^{2}\right) \\
x & =\sum_{i} w_{i} A_{m, i} A_{s, i} e^{-j \varphi_{i}} \\
N & =\sum_{i} w_{i}
\end{aligned}
$$

\section{B. Region Growing}

Instead of just using a predefined search window, region growing starts with using the target pixel as a seed pixel of the region and then iteratively expanding it as follows. For all pixels in the region's border the log-likelihoods $\xi_{\mathrm{LL}}$ of the corresponding patches are computed, and the pixel with the highest similarity is added to the region. We only used $\xi_{\mathrm{LL}}$, as it is less discriminate to changes than $\xi_{\mathrm{KL}}$, resulting in overall more cohesive regions. The border $T$ is defined as all pixels $x$ which are not part of the region $R$, and whose 4 connected neighborhood $N(x)$ adjoins the region, i.e. their intersection is not the empty set:

$$
T=\{x \notin R \mid N(x) \cap R \neq \emptyset\}
$$

This process is repeated until a previously set number of pixels in the region is reached.

Urban areas pose a greater challenge to the region growing based approach due to their highly structured and repetitive but disconnected nature. One possible solution is to use multiple, distributed seed points. For our investigation we generated quasi-random seed points from the Sobol sequence in a large window around the target pixel. Quasi-random or lowdiscrepancy sequences have the advantage over true random sequences that they more evenly cover a selected area.

Overall this leaves us with three nonlocal filter variants to compare

1) the classical NLInSAR filter as described in [2]

2) NLInSARrg with a single seed point, where the static search window of NLInSAR has been replaced with the previously described region growing procedure

3) NLInSARrg with multiple seed points

\section{Simulation}

In order to get an initial estimate of the proposed method's performance a Monte-Carlo simulation was conducted. The simulated terrain consists of a rectangular plateau with jumps in amplitude, phase and coherence.

Figure 2 shows the statistics of the denoised image which is produced by NLInSAR and NLInSARrg with a single seed point. In both cases the patch size was $5 \times 5$ and the search window size $21 \times 21$, meaning the region growing based approach stopped after the size of the region exceeded 441 pixels.

Evidently the NLInSAR approach suffers from higher variance at the edges, since the filter is only able to find a limited number of similar pixels. By replacing the static 

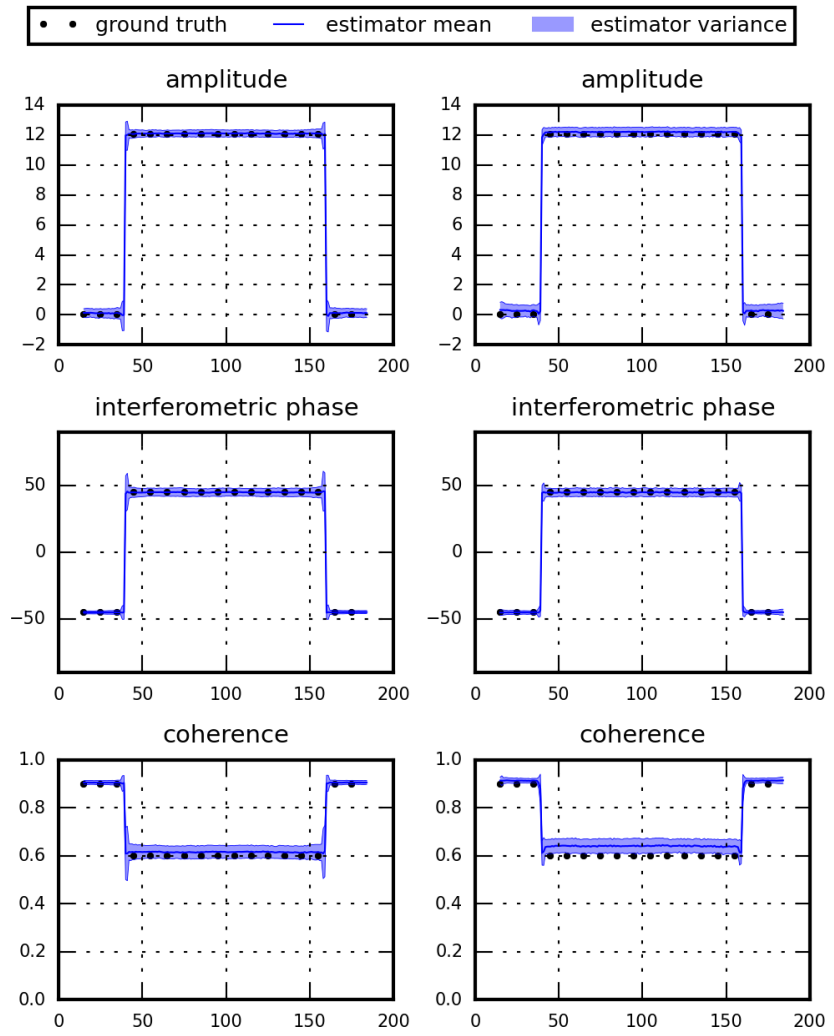

Fig. 2. Comparison of NLInSAR (left) and NLInSARrg with a single seed point (right)

search window with region growing pixels along the edges are discovered, resulting in lower variance.

\section{EXPERIMENTS}

Experiments on real data were conducted for two test sites: an air port near the small town Blatzheim in Germany, and the city Weihai in China.

Figure 3 shows the unfiltered amplitude and interferometric phase images of the test site in Germany plus the results produced by NLInSAR and NLInSARrg with a single seed point. In general the result produced by NLInSAR is smoother and has fewer artifacts in homogeneous areas as can be seen from the landscape surrounding the airport, which consists of forests and agricultural fields. However, NLInSAR misses some details, which is especially apparent in the phase image where the runway is more pronounced for the region growing based approach.

For the urban site we analyzed the effect of multiple seed points. 30 quasi-random seed points were generated in a $41 \times$ 41 window surrounding the target pixel. Figure 4 shows on the left side selected pixels for which the grown regions are depicted on the right side in the middle and last row, for a single and multiple seed points, respectively. Additionally the right side shows the filtering results in the pixels' surrounding areas for NLInSAR, NLInSARrg with a single seed pixel, and NLInSARrg with multiple seed points from top to bottom.
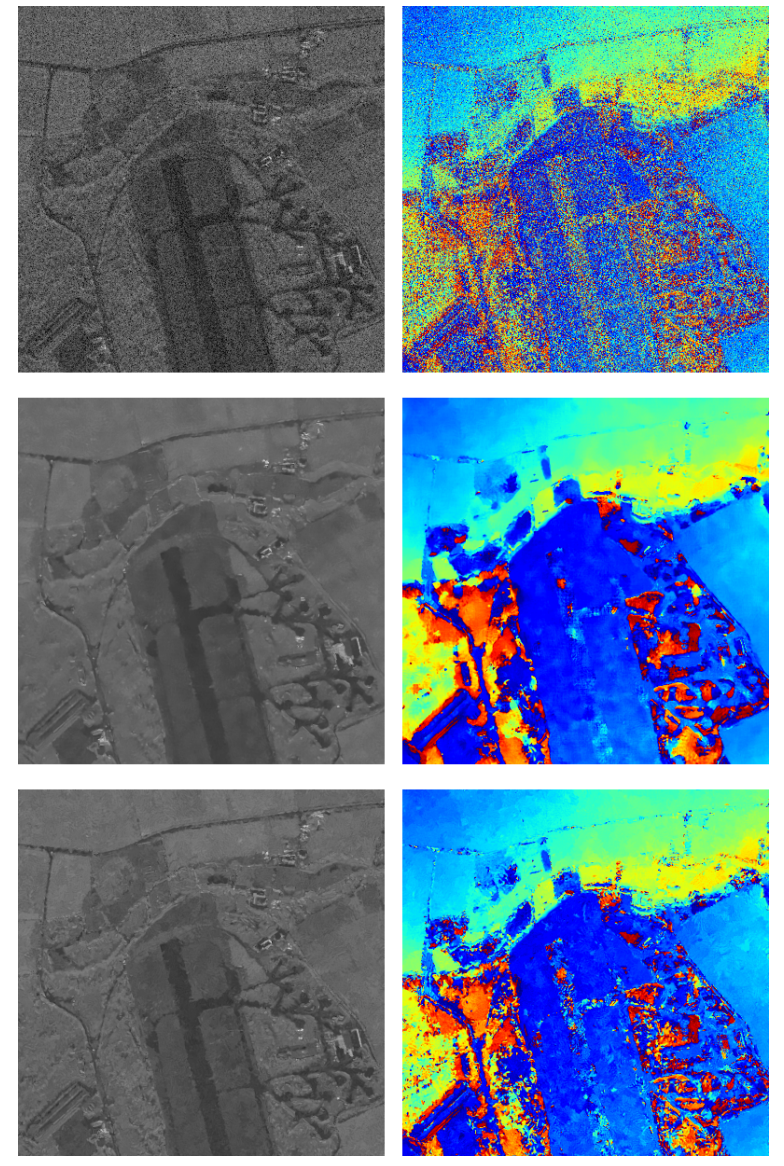

Fig. 3. Test Site Blatzheim; amplitude and interferometric phase from top to bottom: unfiltered data, NLInSAR, NLInSARrg with a single seed point
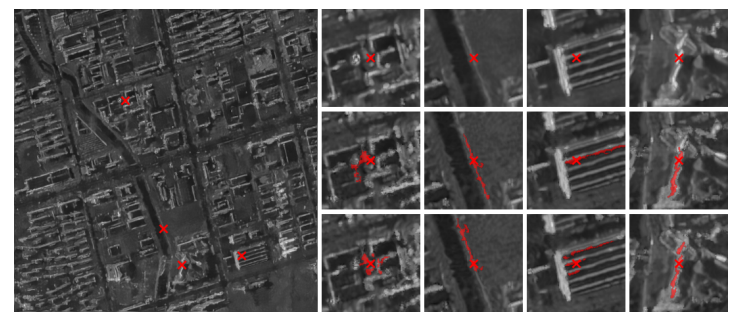

Fig. 4. Test Site Weihai: left side: selected exemplary region growing pixels; right side from top to bottom: filtering results NLInSAR, regions and filtering results for NLInSARrg with a single seed pixel, and with multiple seed pixels.

The denoised results of the complete test site for the three methods are shown in Fig. 5. As with the previous test site the region growing based approaches perform worse on homogeneous areas than NLInSAR, but for heterogeneous areas the output is slightly crisper. Surprisingly using multiple seed points barely enhances the result.

\section{CONCLUSION}

By replacing the fixed search window of a nonlocal filters with an adaptive region growing based window, the filter is able to better resolve fine details, however at the cost of a degraded result for homogeneous areas. These findings where 

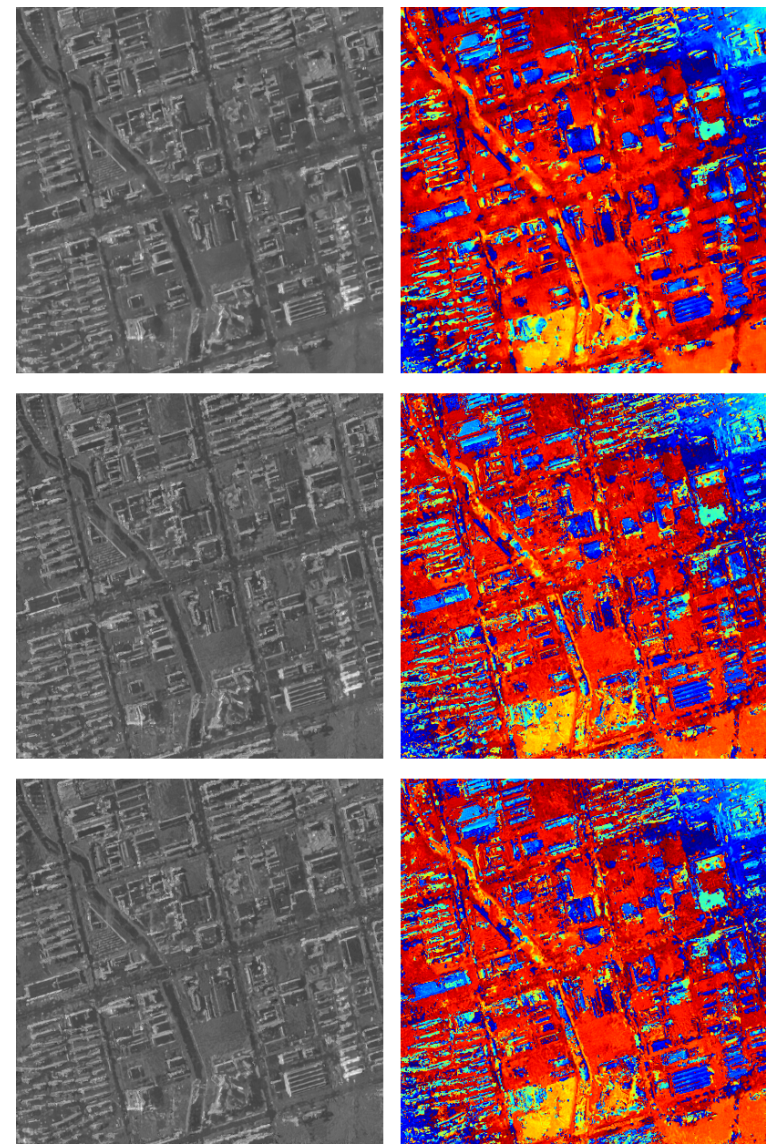

Fig. 5. Test Site Weihai: amplitude and interferometric phase from top to bottom: NLInSAR, NLInSARrg with a single seed point, and NLInSARrg with multiple seed points

confirmed by simulations and tests for rural and urban areas. So far the conventional nonlocal approach is far superior in terms of computational cost to the region growing base approach, as symmetries and previously computed pixel similarities can be more easily exploited [5]. Ways and methods to improve the overall performance of the region growing approach, as well as more dynamic ways of terminating the search for similar pixels to add to the region will be a topic for future investigation. Additionally the applicability to mountainous areas and the resulting fringes will also be part of subsequent research.

\section{REFERENCES}

[1] A. Buades, B. Coll, and J.-M. Morel, "A non-local algorithm for image denoising," in IEEE Computer Society Conference on Computer Vision and Pattern Recognition, 2005. CVPR 2005, vol. 2, Jun. 2005, pp. 60-65 vol. 2.

[2] C.-A. Deledalle, L. Denis, and F. Tupin, "NL-InSAR: Nonlocal Interferogram Estimation," IEEE Transactions on Geoscience and Remote Sensing, vol. 49, no. 4, pp. 1441-1452, Apr. 2011.

[3] X. X. Zhu, R. Bamler, M. Lachaise, F. Adam, Y. Shi, and M. Eineder, "Improving tandem-x dems by non-local insar filtering," in EUSAR 2014; 10th European Conference on Synthetic Aperture Radar; Proceedings of, June 2014, pp. 1-4.

[4] K. Zheng, W. Feng, and H. Chen, "An adaptive non-local means algorithm for image denoising via pixel region growing and merging," in $20103 \mathrm{rd}$

International Congress on Image and Signal Processing (CISP), vol. 2, Oct. 2010, pp. 621-625.

[5] J. Darbon, A. Cunha, T. Chan, S. Osher, and G. Jensen, "Fast nonlocal filtering applied to electron cryomicroscopy," in Biomedical Imaging: From Nano to Macro, 2008. ISBI 2008. 5th IEEE International Symposium on, May 2008, pp. 1331-1334. 